\title{
Dressing versus non-dressing technique for long-term exit-site care in children on continuous ambulatory peritoneal dialysis: a single-center retrospective cohort study
}

\author{
Cahyani Gita Ambarsari, ${ }^{1}$ Eka Laksmi Hidayati, ${ }^{1}$ Lily Mushahar, ${ }^{2}$ Agustina Kadaristiana ${ }^{1}$
}

Check for updates

pISSN: 0853-1773 • elSSN: 2252-8083 https://doi.org/10.13181/mji.oa.204171 Med J Indones. 2020;29:290-7

Received: August 30, 2019

Accepted: August 08, 2020

Authors' affiliations:

${ }^{1}$ Department of Child Health, Faculty of Medicine, Universitas Indonesia, Cipto Mangunkusumo Hospital, Jakarta, Indonesia, ${ }^{2}$ Department of Nephrology, Tuanku Ja'afar Hospital, Seremban, Negeri Sembilan, Malaysia

\section{Corresponding author:}

Cahyani Gita Ambarsari

Department of Child Health, Faculty of Medicine, Universitas Indonesia, Cipto Mangunkusumo Hospital, Pediatric Nephrology Division, URJT Building $5^{\text {th }}$ Floor, Jalan Diponegoro No. 71, Central Jakarta 10430, DKI Jakarta, Indonesia Tel/Fax: +62-21-3907742/

+62-21-3907743

E-mail: cahyani.ambarsari@ui.ac.id/ cahyanigita@gmail.com

\begin{abstract}
BACKGROUND There is no consensus on the optimal long-term exit-site care strategy in children on long-term peritoneal dialysis (PD) worldwide. Thus, this study aimed to compared the dressing versus non-dressing technique for long-term exit-site care to prevent PD-related infection.
\end{abstract}

METHODS This retrospective cohort study involved patients aged $<18$ years with end-stage kidney disease who were on continuous ambulatory PD at the Cipto Mangunkusumo Hospital between March 2014 and March 2019. Long-term exit-site care was initiated within 3 months after the insertion of Tenckhoff catheter. The patients and caregivers can choose to either maintain the dressing method or change to the non-dressing method for the subsequent long-term exit-site care. The follow-up was performed until the following 6 months.

RESULTS Out of 34 patients, 18 were treated without dressing and 16 with dressing technique. The peritonitis rates were 0.17 and 0.06 episodes per year at risk in the non-dressing and dressing groups; the adjusted incidence rate ratio was 0.4 ( $95 \%$ confidence interval $=0.04-3.25 ; p=0.4)$. Allergic contact dermatitis occurred in $15 / 18$ (83\%) patients in the non-dressing group and $11 / 16(69 \%)$ in the dressing group before long-term exit-site care was applied. Ultimately, no patient in the non-dressing group developed allergic contact dermatitis, whereas 11 (69\%) had allergic contact dermatitis in the dressing group at the end of follow-up.

CONCLUSIONS Non-dressing technique may increase the risk of peritonitis in children on long-term PD; however, it is beneficial for children vulnerable to allergic contact dermatitis.

KEYWORDS child, contact dermatitis, end-stage kidney disease, infection, peritonitis
Recently, peritoneal dialysis (PD) is the preferred dialysis modality among pediatric endstage kidney disease (ESKD) patients in the Pediatric Dialysis Unit, Cipto Mangunkusumo Hospital. However, the complications of PD are inevitable. Noninfectious complications, such as fluid overload and pleuroperitoneal fistula, were reported in the Pediatric Dialysis Unit, Cipto Mangunkusumo Hospital.1,2 PD catheter-related infection (exit-site infection [ESI] and tunnel infection) is a risk factor for peritonitis, a major cause of technical failure within a year of PD initiation. ${ }^{3,4}$ A recently published case report provides a good example that conversion to PD early will improve the quality of life, convenience to the patient and caregivers, reduce the complication rate, including infections. ${ }^{5}$ Therefore, prevention of PD catheter-related infection is essential to ensure the continuity of PD. Some preventive measures 
include the use of prophylactic antibiotics before the insertion of PD catheter and application of daily topical antibiotic care on the catheter exit-site. ${ }^{6}$

Unfortunately, robust studies on long-term exitsite care are still extremely limited, particularly in the pediatric population. Based on the available studies, the International Society of Peritoneal Dialysis (ISPD) recommends maintenance of dressing for up to a week following catheter insertion to prevent exitsite trauma during early exit-site care. Subsequently, the dressing is changed after 1 week using a sterile technique until the healing of the exit-site wound, which is characterized by a normal skin appearance surrounding the exit-site without gaping. ${ }^{7}$ For longterm exit-site care for pediatric patients, the use of antiseptic solution and topical antibiotic is still recommended in spite of limited evidence, which is largely obtained from retrospective studies. ${ }^{7}$ No study showed that a particular type of cleansing agent is superior to others for infection prevention. ${ }^{6}$ There is no consensus on the use of dressing or the selection of the dressing type for patients with longterm PD. Gauze dressing is usually used to maintain the exit-site clean, protect the wound against trauma, and support the catheter. ${ }^{6,8}$ However, in a randomized controlled pilot trial conducted in adult PD patients, cleaning the exit-site after shower, followed by the application of topical antibiotics without antiseptic agent or dressing was found to be adequate. ${ }^{9}$ The advantage of non-dressing procedure is that it may prevent the development of allergic contact dermatitis in response to the antiseptic liquid or the dressing film. Moreover, the non-dressing technique may also reduce the use of disposables and save time for changing the dressing.

Studies have shown that the occurrence of noninfectious skin lesions due to dressing or antiseptic solution is relatively rare. ${ }^{10,11}$ However, we have found allergic contact dermatitis quite frequent in pediatric patients on long-term PD at the Pediatric Dialysis Unit, Cipto Mangunkusumo Hospital. Previously published studies on the dressing technique on long-term exit-site care have always been conducted in adults. Therefore, this study aimed to compare the effectiveness of non-dressing versus dressing technique for longterm exit-site care with respect to the prevention of PD catheter-related infections in pediatric PD population.

\section{METHODS}

This was a retrospective cohort study. The inclusion criteria were children aged $<18$ years with ESKD who were on continuous ambulatory PD treatment for at least 9 months at the Cipto Mangunkusumo Hospital between March 2014 and March 2019. Institutional review board approval was obtained from the Ethics Committee of the Faculty of Medicine, Universitas Indonesia (KET-565/UN2.F1/ ETIK/PPM.00.02/2019). Data about demographic and clinical variables were obtained from the medical records. Patients who had exposed external cuff, dropped out due to various reasons before the opening of the early exit-site dressing (due to death, transfer to adult care unit, or loss to follow-up), followed up for less than 3 months after catheter insertion, and incomplete medical records were excluded. In the beginning of the study, there were 18 subjects who underwent Staphylococcus aureus screening. In daily practice, this is not an obligatory protocol, and its implementation relies on the doctor's preference.

Early exit-site dressing is the use of dressing following the insertion of PD catheter until the exitsite wound is completely healed according to the ISPD recommended criteria.7 At the Cipto Mangunkusumo Hospital, dressing after catheter insertion is maintained until 1-2 weeks following catheter insertion. Subsequently, the dressing is typically changed once daily for 3 months to ensure complete healing at the exit-site. After 3 months, the dressing might be maintained depending on the patients' or caregivers' preferences; however, we recommend the non-dressing protocol, especially for patients who experience allergic contact dermatitis to the dressing.

All patients must clean the exit-site once daily using antibacterial soap during their bath and subsequently dry it using a clean towel by one particular caregiver. After the site had dried up, patients using dressing were asked to clean their exit-site with $2 \%$ chlorhexidine solution, apply gentamicin ointment, cover the exitsite using sterile gauze, and secure the catheter using hypafix ${ }^{\circledR}$ (BSN medical, Germany), tegaderm ${ }^{\circledR}\left(3 \mathrm{M}^{\mathrm{TM}}{ }^{\mathrm{M}}\right.$, Germany), or micropore ${ }^{\circledR}\left(3 \mathrm{M}^{\mathrm{TM}}\right)$ surgical tape. For patients who did not use the dressing, the patients were only asked to apply gentamicin ointment in the surrounding of the exit-site and secure the catheter using surgical tape after the site had dried up. Follow-up 
period started from 3 months after catheter insertion until the following 12 months. For patients with severe dermatitis, the exit-site should be opened before 3 months; however, the follow-up was still performed within 3 months after catheter insertion. Patients were subsequently followed up every month until the following 12 months. Only one investigator followed up the exit-site to avoid misclassification bias.

Parents' educational degree, socioeconomic background, and patients' hygiene standard were the confounding factors of this study. History of dermatitis, albumin levels, and potassium levels were the potential effect modifiers. The exposure, as well as the predictor of the study, is the application of nondressing technique. The primary outcome was ESI, and the secondary outcomes were tunnel infection and peritonitis. ESI was defined as the presence of purulent discharge with or without skin erythema at the catheter-epidermal interface. Tunnel infection was defined as the presence of inflammatory features along the catheter tunnel. ${ }^{6}$ The diagnosis of peritonitis was established when there were at least two of the following signs: (1) clinical symptoms consistent with peritonitis such as abdominal pain and/or dialysis effluent, (2) dialysis effluent white blood cell count of $>100 / \mu$ l or $>0.1 \times 10^{9} / \mathrm{l}$ after a dwell time of at least 2 hours with $>50 \%$ polymorphonuclear leukocytes, and (3) positive dialysis effluent culture. ${ }^{12}$ Peritonitis, ESI, and tunnel infection rate were presented as episode per year at risk and episode per patientmonth, as recommended in the ISPD guidelines. ${ }^{12,13}$ Allergic contact dermatitis was diagnosed based on clinical manifestations of dermatitis (polymorphic cutaneous inflammatory features such as erythema and vesiculation in the acute phase or dryness, lichenification, and fissure in the chronic phase) and history of contact with exogenous agent. ${ }^{14}$
Statistical analysis was performed using SPSS software version 24 (IBM Corp., USA). Continuous variables were reported as median and interquartile range, and categorical variables as proportion and percentage. Depending on the normality and homogeneity of variance, continuous data were compared using student $t$-test, assuming that there was equal variance. Moreover, Mann-Whitney $U$ test was used in case of unequal variance, and categorical data were compared using the chi-square test. If the assumption for chi-square was not met, Fischer's exact test was used. Incidence rates in both groups were compared using the z-score test. Multivariable incidence rate was analyzed using a negative binomial distribution since the assumption of Poisson regression was not met. Statistically significant difference considered if $p$-value $<0.05$.

\section{RESULTS}

A total of 41 patients had been selected for the study, but only 34 were included (Figure 1). There were 18 patients with non-dressing technique (Figure 2a) and 16 with dressing technique (Figure $2 b$ ) for the long-term exit-site care. Bilateral kidney hypoplasia is the most prevalent primary kidney disease underlying the ESKD in both groups (Table 1). Screening for bacterial colonization was performed in three locations before inserting the PD catheter (anterior nares, axilla, and groin) in 18 patients. None of the patients who underwent screening had colonization of S. aureus.

All patients had the double-cuffed straight Tenckhoff catheter, and the majority had it in a lateral direction. Allergic contact dermatitis on the exit-site was relatively common (Figure 2c); usually, the patients were allergic to surgical tapes (such as
Figure 1. Schematic illustration of the study design and patientselection criteria. HD=hemodialysis
Non-dressing $(n=18)$
Exit-site dressing $(n=16)$ 

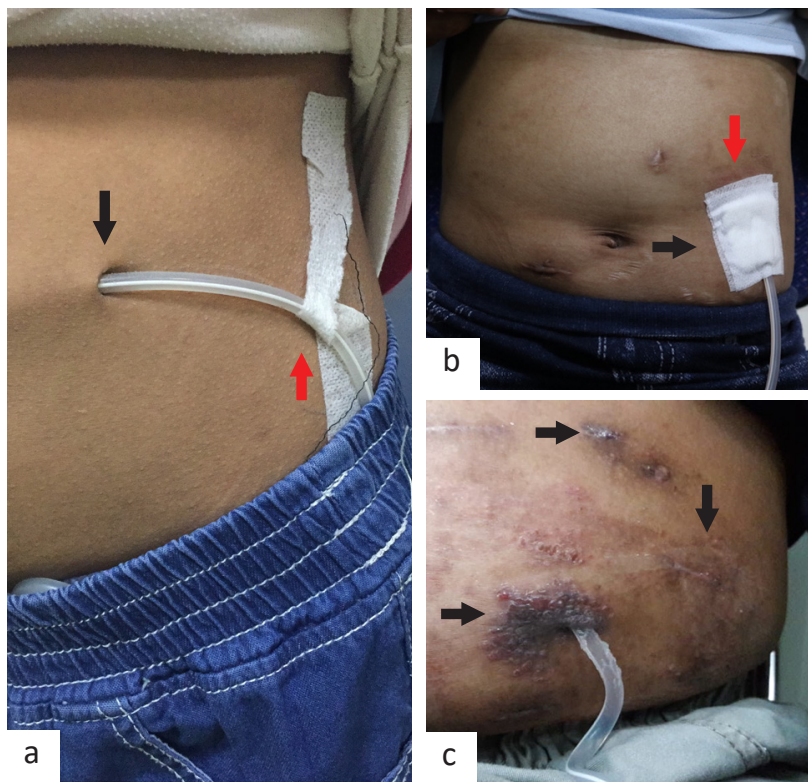

Figure 2. (a) Exit-site care with non-dressing technique (black arrow) and peritoneal dialysis (PD) catheter fixation with tape (red arrow); (b) exit-site care with dressing technique (black arrow) and dermatitis-risk zone (red arrow); and (c) allergic contact dermatitis due to dressing technique (black arrows) hypafix ${ }^{\circledR}[B S N$ medical $]$, tegaderm ${ }^{\circledR}\left[3 M^{T M}\right]$, and micropore ${ }^{\circledR}\left[3 \mathrm{M}^{\mathrm{TM}}\right]$ ) or to antiseptic solution (such as $2 \%$ chlorhexidine). Serum albumin or potassium levels were similar in both groups.

PD-related infections are shown in Table 2. There was one episode of tunnel infection in the dressing group. Peritonitis episodes were more frequent in the non-dressing group than in the dressing group (Table 2 ). Both patients with peritonitis were responsive to empirical antibiotic therapy, which was intraperitoneal gentamicin.

There was one episode of tunnel infection in the dressing group with a tunnel infection rate of 0.13 episodes per year at risk. There were similar characteristics of tunnel infection between the nondressing and dressing groups. Unlike the tunnel infection, peritonitis episodes in the non-dressing group were more common than in the dressing group. Three episodes of peritonitis occurred in three patients in the non-dressing group ( 0.17 episodes per year at risk) than in the dressing group ( 0.06 episodes

Table 1. Patient characteristics

\begin{tabular}{|lccc}
\hline Variable & $\begin{array}{c}\text { Non-dressing, } \\
\mathrm{n}(\%)(\mathrm{N}=18)\end{array}$ & $\begin{array}{c}\text { Dressing, } \\
\mathrm{n}(\%)(\mathrm{N}=16)\end{array}$ & $p$ \\
\hline Age (year), median (IQR) & $12.9(3.6)$ & $12.4(5.2)$ & $0.9^{*}$ \\
\hline Male sex & $12(67)$ & $10(63)$ & $0.8^{+}$ \\
\hline Primary cause & & & - \\
\hline Bilateral kidney hypoplasia & $11(61)$ & $7(44)$ & \\
\hline APSGN & $0(0)$ & $1(6)$ & \\
\hline Nephrotic syndrome & $4(22)$ & $3(19)$ & \\
\hline Neurogenic bladder & $0(0)$ & $3(19)$ & $0.8^{*}$ \\
\hline Lupus nephritis & $2(11)$ & $0(0)$ & \\
\hline Inherited hypercalciuria & $1(6)$ & $0(0)$ & $0.5^{*}$ \\
\hline VUR & $0(0)$ & $4(25)$ & $0.5^{*}$ \\
\hline Catheter orientation & & & \\
\hline Lateral & $17(94)$ & $11(69)$ & $1^{*}$ \\
\hline Downward & $1(6)$ & $5(31)$ & \\
\hline Screening swab & $9(50)$ & $9(56)$ & $13(81)$ \\
\hline Prophylaxis antibiotic & $14(82)$ & $3.4(0.8)$ & \\
\hline Allergic contact dermatitis & $15(83)$ & $3.5(0.9)$ & \\
\hline Before long-term exit-site protocol & $0(0)$ & $11(69)$ & \\
\hline After long-term exit-site protocol & $3.2(0.9)$ & & \\
\hline Albumin (g/dl), median (IQR) & $3.6(1.1)$ & & \\
\hline Potassium (mEq/I), median (IQR) & & & \\
\hline & & & \\
\hline
\end{tabular}

IQR=interquartile range; APSGN=acute post-streptococcal glomerulonephritis; VUR=vesicoureteral reflux *Mann-Whitney U test; 'Fisher's exact test 
Table 2. PD-related infections

\begin{tabular}{lcc}
\hline Variable & Non-dressing, $\mathrm{n}(\mathrm{N}=18)$ & Dressing, $\mathrm{n}(\mathrm{N}=16)$ \\
\hline Patient with ESI & 0 & 0 \\
ESI episode & 0 & 0 \\
\hline ESI rate & 0 & 0 \\
$\quad$ Episode per year at risk & 0 & 0 \\
\hline Episode per patient-month & $\mathrm{NA}$ & $\mathrm{NA}$ \\
\hline ESI organism & 0 & 1 \\
\hline Patient with tunnel infection & 0 & 1 \\
\hline Tunnel infection episode & & \\
\hline Tunnel infection rate & 0 & 0.13 \\
\hline Episode per year at risk & 0 & $1: 96$ \\
\hline Episode per patient-month & 3 & 1 \\
\hline Patient with peritonitis & 3 & 1 \\
\hline Peritonitis episode & & \\
\hline Peritonitis rate & 0.17 & 0.06 \\
\hline Episode per year at risk & $1: 72$ & $1: 192$ \\
\hline Episode per patient-month & & 0 \\
\hline Peritonitis pathogen & 1 & 0 \\
\hline Escherichia coli & 1 & 0 \\
\hline Acinetobacter baumannii & 1 & 1 \\
\hline Staphylococcus aureus & 0 & \\
\hline Staphylococcus alpha hemolytic & & \\
\hline
\end{tabular}

$\mathrm{PD}=$ peritoneal dialysis; $\mathrm{ESI}=$ exit-site infection; $\mathrm{NA}=$ not applicable

per year at risk). The time-to-first peritonitis in the non-dressing group were 5.9, 0.7, and 7.7 months, and in the dressing group was 9.1 months. The incidence rate difference was 0.4 (95\% confidence interval $[\mathrm{Cl}]$ $=0.04-3.25 ; p=0.4)$ for peritonitis and $-1.25(95 \% \mathrm{Cl}=$ -3.7-1.2; $p=0.47$ ) for tunnel infection.

Among all three patients who had tunnel infection and peritonitis, only one who experienced peritonitis had undergone screening for $S$. aureus colonization before PD catheter insertion and had shown negative results; however, one patient indeed experienced tunnel infection due to $S$. aureus. The culture results on peritonitis in the non-dressing group showed that the Gram-negative bacteria identified on culture was Acinetobacter baumannii. The result of one sterile bacterial culture in the non-dressing group might be attributable to technical error (during sample collection).

\section{DISCUSSION}

Cipto Mangunkusumo Hospital has started the pediatric PD program since 2014 as one option of kidney replacement therapy. ${ }^{1}$ The majority of PD units including our unit have been using the dressing technique as a method for long-term PD exit-site care. In a survey conducted in Austria, only 14 of 332 (4\%) PD patients did not use the dressing technique for their long-term exit-site care. ${ }^{8}$ The inclination to dressing technique is partly because there has not been sufficient number of studies, particularly longterm randomized clinical trials (RCTs), regarding the non-dressing technique. However, the available study investigating the efficacy of non-dressing technique showed a satisfactory outcome. ${ }^{9}$ The study suggested that the procedure is more practical and costeffective as it minimizes the use of disposables. ${ }^{9}$ More importantly, the non-dressing technique is as effective and safe as the dressing technique in preventing PDrelated infection. ${ }^{2}$ However, our findings did not suggest the superiority of the non-dressing technique to prevent PD-related infection compared with that of the dressing technique, in contrast with the results from the adult population study.

In our study, the peritonitis rate in the non-dressing group was higher than in the dressing group. Until now, the ISPD has not determined the minimal target for ESI and tunnel infection rate due to the lack of adequate 
evidence. ${ }^{6}$ Nevertheless, we did not find any episode of ESI in the non-dressing group, resulting in ESI rate that was lower than that reported among patients in the USA ( 0.25 episodes per year at risk). ${ }^{15}$ Peritonitis rate in the non-dressing group was higher than in the dressing group. However, the peritonitis rate was much better than the ISPD recommendation, which is 0.5 episodes per year at risk. $^{12}$ A recently published study from our center reported that between 2014 and 2019, our mean time-to-first peritonitis in all patients was 6.6 months ( $95 \% \mathrm{Cl}=2.7-10.4$ ) following the initiation of PD. ${ }^{1}$ Our current findings revealed that the time-to-first peritonitis in the non-dressing group were 0.7 months and 5.9 months, suggesting that peritonitis in the nondressing group occurred earlier than 6.6 months. This might be related with the fact that patients who were advised to implement the non-dressing technique were cases who had dermatitis before. In children with chronic kidney disease (CKD), disturbed skin barrier is an important concern, and this problem intensifies as the disease progresses. Most CKD children reported dry skin, and the problem of xerosis was more frequent in patients on dialysis (67.6\%) than on conservative treatment (42.1\%; $p=0.01$ ), with patients undergoing PD having less skin moisture than those undergoing hemodialysis. ${ }^{16}$ The $\mathrm{pH}$ of the stratum corneum is increased in dialysis patients, causing an interruption in protease activation and, consequently, resulting in exfoliation. ${ }^{16}$ For this reason, we consider that children in the non-dressing group have disturbed skin barrier causing them to be prone to infections.

In our center, for older children, applying the nondressing protocol is practical as they can perform longterm exit-site care by themselves without depending on their caregivers. In contrast, the dressing protocol can only be performed with assistance from the caregivers, even in the case of older children. Assistance from the caregivers is required for changing the dressing, applying the adhesive tape, and ensuring that all sides of the dressing are well covered.

The application of dressing technique for longterm exit-site care is widely presumed as the best way to prevent contamination at the exit-site. Therefore, patients tend to feel reluctant to take off their wound dressing because of feeling insecure. ${ }^{8}$ Several studies have demonstrated higher rates of PD-related infection in the non-dressing group than in the dressing group. ${ }^{17,18}$ However, studies that support the dressing technique may have some limitations as there are different procedures for preventing PD-related infection that were performed in the past and in the current situation. For example, prophylactic topical antibiotics were not applied in both studies.

The results of our study are also different from a recent RCT conducted by Mushahar et al. ${ }^{9}$ In the RCT that involved 97 adult patients, subjects in the non-dressing group were only asked to apply topical mupirocin after cleaning the exit-site, whereas subjects in the dressing group were asked to cover the exitsite with sterile gauze after cleansing with povidone iodine solution and applying topical mupirocin. Results showed that non-dressing technique did not increase the risk of ESI, and the median time-to-first peritonitis was significantly longer in the non-dressing group.

A study conducted in a smaller sample of adult patients also showed the efficacy and safety of nondressing technique concerning ESI and peritonitis prevention. ${ }^{19}$ The discrepancy between the results of both previous studies and our study may be attributed to the different patient characteristics, since our patients experienced a high incidence of dermatitis. Some risk factors that may increase PD catheterrelated infections, particularly peritonitis, include poor patient compliance, upward exit-site orientation, and touch contamination. ${ }^{20}$ In our study, we did not confirm certain risk factors for peritonitis and tunnel infection. The nasal carriage of $S$. aureus is the main risk factor for ESI and tunnel infection. Therefore, the ISPD recommends screening for nasal carriage of S. aureus before insertion of the Tenckhoff catheter. ${ }^{6}$ In our unit, screening for S. aureus colonization was performed only for some patients. Out of three patients who experienced tunnel infection and peritonitis, only one underwent a screening test for S. aureus colonization with a negative result. Moreover, all patients who experienced peritonitis were not screened test for S. aureus colonization.

Although there was no bacterial colonization before the insertion of Tenckhoff catheter, colonization may have occurred after the patient experienced allergic contact dermatitis due to dressing material. A 2013 study in Korea showed that S. aureus colonization in the skin of patients with atopic dermatitis was more common than in those without any skin lesions, and the colonization rate was more elevated in acute lesions of atopic dermatitis. ${ }^{21}$ At Cipto Mangunkusumo Hospital, we recommend taking off the dressing for patients with severe dermatitis. This may also have affected the 
peritonitis rate, which was found to be higher in the non-dressing group. The lack of compliance concerning hand hygiene and connection technique may have also affected the incidence of infection. Unfortunately, data on patient compliance were unavailable at Pediatric Dialysis Unit, Cipto Mangunkusumo Hospital. Only one case was reported to have recurrent peritonitis due to poor compliance to the connection technique. ${ }^{22}$

Daily use of topical mupirocin has also been shown to be effective in preventing ESI due to $S$. aureus, and it has been recommended by the ISPD. ${ }^{6}$ However, in our unit, we prefer topical gentamicin over topical mupirocin based on unpublished data about the pattern of microorganisms that cause ESI, tunnel infection, and peritonitis in children on long-term PD at the pediatric dialysis unit of Cipto Mangunkusumo Hospital. The data demonstrated that $S$. aureus is not the main cause of PD-related infection. Moreover, the number of nasal carriers of $S$. aureus among pediatric patients on PD at the Pediatric Dialysis Unit at the Cipto Mangunkusumo Hospital is extremely low. Both gentamicin and mupirocin are effective in preventing Gram-positive bacterial infection; however, gentamicin offers a greater advantage in preventing Gram-negative bacterial infection.23 In addition, as a developing country, Indonesia often experience insufficiency of medical resources including medication and laboratory support. Since topical gentamicin is more affordable and accessible than topical mupirocin in Indonesia, we decided to use it to our patients.

Long-term exit-site care in children who experienced dermatitis has become a unique challenge. Some previous studies suggest that noninfectious lesions rarely occur in patients on PD. ${ }^{24}$ However, in Cipto Mangunkusumo Hospital, the incidence of dermatitis in pediatric patients may reach as high as $73 \%$ (27/37); of these, 15 were in the non-dressing group and 12 in the dressing group. Most lesions were allergic contact dermatitis caused by surgical tape. The recommended treatment for contact dermatitis includes the elimination of the causative agent and topical application of steroids and barrier cream. ${ }^{24}$ In the Pediatric Dialysis Unit, Cipto Mangunkusumo Hospital, non-dressing care is recommended for all patients who experience allergic contact dermatitis at the exit-site. The non-dressing technique eliminates the problem of dermatitis; moreover, it is cost-efficient and more practical for daily application. When the patients use the nondressing technique, their compliance on procedures for preventing infection such as hand hygiene and connection technique must be seriously taken into concern. However, some patients or their caregivers opted for dressing care. After the follow-up period, all dermatitis cases resolved in the non-dressing group, whereas the dermatitis rate did not change in the dressing group.

Our study is the first to evaluate the effect of non-dressing technique for long-term exit-site care in pediatric PD patients after the study by Watson et al ${ }^{18}$ conducted 30 years ago. We also found noninfectious lesions as one of the side effects of PD. Some limitations of our study should be acknowledged. This was a retrospective, single-center study with a small patient sample size; therefore, our results may have been affected by information bias. Patch test examination to diagnose allergic contact dermatitis was not routinely performed. Moreover, some risk factors could not be evaluated due to the small sample size. The duration of follow-up for each patient was also different. However, we made some efforts to minimize bias including adjusting for follow-up duration and variables that are strongly assumed to affect the outcome in evaluating the incidence rate ratio. A prospective RCT with larger sample size is necessary to provide more definitive evidence of the effect of non-dressing technique in pediatric patients.

In conclusion, the selection of dressing or nondressing procedure for pediatric patients should be considered carefully. Despite the relatively low rate of peritonitis, no ESI and tunnel infection were found in the non-dressing group. Therefore, our study suggests that non-dressing technique may increase the risk of peritonitis in children on long-term PD. Nevertheless, although the non-dressing technique has not been proven to be better than the dressing technique, it seems that its application is useful for patients with allergic contact dermatitis.

Conflict of Interest

The authors affirm no conflict of interest in this study.

\section{Acknowledgment}

The authors would like to thank Professor Taralan Tambunan, Professor Partini Pudjiastuti Trihono, Sudung Oloan Pardede, MD, $\mathrm{PhD}$, and Henny Adriani Puspitasari, MD for their care of the patients in the Department of Child Health, Cipto Mangunkusumo Hospital. We also thank Elysabeth Muliawan, MD and Angela Grace, MD for their editing service on the English version of this manuscript. We kindly acknowledge the pediatric dialysis nurses of Cipto Mangunkusumo Hospital: Yetti Marlina, Mustaqimah, Dorkasturi Sitompul, Meldahania, 
Ani Suryani, Emma Meida Ekawati, and Resti Fitria Pramandani in providing information and maintaining the database.

\section{Funding Sources}

None.

\section{REFERENCES}

1. Ambarsari CG, Trihono PP, Kadaristiana A, Tambunan T, Mushahar L, Puspitasari HA, et al. Five-year experience of continuous ambulatory peritoneal dialysis in children: a single center experience in a developing country. Med J Indones. 2019;28(4):329-37.

2. Ambarsari CG, Bermanshah EK, Putra MA, Rahman FHF, Pardede SO. Effective management of peritoneal dialysis-associated hydrothorax in a child: a case report. Case Rep Nephrol Dial. 2020;10(1):18-25.

3. See EJ, Johnson DW, Hawley CM, Pascoe EM, Badve SV, Boudville N, et al. Risk predictors and causes of technique failure within the first year of peritoneal dialysis: an Australia and New Zealand Dialysis and Transplant Registry (ANZDATA) Study. Am J Kidney Dis. 2018;72(2):188-97.

4. van Diepen AT, Jassal SV. A qualitative systematic review of the literature supporting a causal relationship between exit-site infection and subsequent peritonitis in patients with end-stage renal disease treated with peritoneal dialysis. Perit Dial Int. 2013;33(6):604-10.

5. Ambarsari CG, Cahyadi D, Sari L, Satria O, Sahli F, Darmadi TL, et al. Late diagnosis of Lesch-Nyhan disease complicated with end-stage renal disease and tophi burst: a case report. Ren Fail. 2020;42(1):113-21.

6. Szeto CC, Li PK, Johnson DW, Bernardini J, Dong J, Figueiredo $A E$, et al. ISPD -trgcatheter-related infection recommendations: 2017 update. Perit Dial Int. 2017;37(2):141-54.

7. Warady BA, Bakkaloglu S, Newland J, Cantwell M, Verrina E, Neu $A$, et al. Consensus guidelines for the prevention and treatment of catheter-related infections and peritonitis in pediatric patients receiving peritoneal dialysis: 2012 update. Perit Dial Int 2012;32(Suppl 2):S32-86.

8. Kopriva-Altfarht $G$, Konig P, Mundle M, Prischl F, Roob JM, Wiesholzer $M$, et al. Exit-site care in Austrian peritoneal dialysis centers-A nationwide survey. Perit Dial Int. 2009;29:330-9.

9. Mushahar L, Mei LW, Yusuf WS, Sivathasan S, Kamaruddin N, Idzham NJ. Exit-site dressing and infection in peritoneal dialysis: a randomized controlled pilot trial. Perit Dial Int. 2016;36(2):13539.

10. Schmitt R, Haller H, Hiss M. Erythematous rash around peritoneal dialysis catheter exit site. Am J Kidney Dis. 2012;60:A29-31.

11. Chasset F, Pecquet $C$, Cury K, Sesé L, Moguelet P, Francès $C$, et al.
Bullous rash around a peritoneal dialysis catheter exit site. Ann Dermatol Venereol. 2015;142(6-7):438-42. French.

12. Li PK, Szeto CC, Piraino B, de Arteaga J, Fan S, Figueiredo $\mathrm{AE}$, et al. ISPD peritonitis recommendations: 2016 update on prevention and treatment. Perit Dial Int. 2016;36(5):481-508.

13. Li PK, Szeto CC, Piraino B, Bernardini J, Figueiredo AE, Gupta A, et al. Peritoneal dialysis-related infections recommendations: 2010 update. Perit Dial Int. 2010;30(4):393-423.

14. Johnston GA, Exton LS, Mohd Mustapa MF, Slack JA, Coulson IH, English JS, et al. British Association of Dermatologists' guidelines for the management of contact dermatitis 2017. $\mathrm{Br} J$ Dermatol 2017;176(2):317-29.

15. Swartz SJ, Neu A, Mason AS, Richardson T, Rodean J, Lawlor $J$, et al. Exit site and tunnel infections in children on chronic peritoneal dialysis: findings from the Standardizing Care to Improve Outcomes in Pediatric End Stage Renal Disease (SCOPE) Collaborative. Pediatr Nephrol. 2018;33(6):1029-35.

16. Wojtowicz-Prus E, Kilis-Pstrusinska K, Reich A, Zachwieja K, Miklaszewska M, Szczepanska M, et al. Disturbed skin barrier in children with chronic kidney disease. Pediatr Nephrol. 2015;30:333-8.

17. Luzar MA, Brown CB, Balf D, Hill L, Issad B, Monnier B, et al. Exit-site care and exit-site infection in continuous ambulatory peritoneal dialysis (CAPD): results of a randomized multicenter trial. Perit Dial Int. 1990;10(1):25-9.

18. Watson AR, Vigneux A, Hardy BE, Balfe JW. Six-year experience with CAPD catheters in children. Perit Dial Int. 1985;5(2):119-22.

19. Taheri S, Ahmadnia M, Mortazavi M, Karimi S, Reihani H, Seirafian S. Comparing the effect of dressing versus no-dressing on exit site infection and peritonitis in chronic ambulatory peritoneal dialysis patients. Adv Biomed Res. 2017;6:5.

20. Sethna CB, Bryant K, Munshi R, Warady BA, Richardson T, Lawlor $J$, et al. Risk factors for and outcomes of catheter-associated peritonitis in children: The SCOPE Collaborative. Clin J Am Soc Nephrol. 2016;11(9):1590-6.

21. Park HY, Kim CR, Huh IS, Jung MY, Seo EY, Park JH, et al. Staphylococcus aureus colonization in acute and chronic skin lesions of patients with atopic dermatitis. Ann Dermatol. 2013;25(4):410-6.

22. Ambarsari CG, Rahman FH, Bermanshah EK, Kadaristiana A. An unusual case of peritoneal dialysis twisted catheter in a child. Madjalah Kedokt Indones. 2020;70(2):27-31.

23. Tsai CC, Yang PS, Liu CL, Wu CJ, Hsu YC, Cheng SP. Comparison of topical mupirocin and gentamicin in the prevention of peritoneal dialysis-related infections: a systematic review and meta-analysis. Am J Surg. 2018;215(1):179-85.

24. Siddiqui M, Bradford L, Kaley J, Johnson G, Kim KH, Addis K, Singh M. Noninfectious peritoneal dialysis exit site rash-an unusual case report and review of the literature. Kidney Int Rep. 2018;3(1):11-3. 